Bull. Mater. Sci., Vol. 17, No. 6, November 1994, pp. 863-873. (9 Printed in India.

\title{
The mechanical response of tetragonal zirconia polycrystal to conical indentation
}

\author{
$S$ A SYED ASIF and S K BISWAS \\ Department of Mechanical Engineering, Indian Institute of Science, Bangalore 560012 , \\ India
}

\begin{abstract}
Blocks of 3Y-TZP were indented with conical diamond indenters. Indentation caused tetragonal to monoclinic phase transformation in a subsurface. Of the cracks generated in the subsurface, radial and lateral cracks can be accounted for by a continuum model of the indented subsurface, built using a combination of the Boussinesq and blister stress fields. Additional ring, median and cone cracks were also observed. It is hypothesized that the latter are motivated by the reduction in blister strength or residual energy brought about by the material damage caused by the phase transformation. This damage reduces the load bearing capacity of the material progressively with increasing normal load.
\end{abstract}

Keywords. TZP; conical indentation.

\section{Introduction}

Metastable tetragonal zirconia-based ceramics have attracted attention in engineering because of their ability to undergo stress-induced transformation toughening. The effect of dialational and shear stresses on transformation plasticity of these materials have been discussed by Chen and Reyes-Morel (1986). In this and other (Marshall and James 1986; Martinez-Fernandes et al 1991) studies indentation by a rigid indentor has often been used to induce transformation and to note the resulting mechanical response of the material.

The present paper is concerned with the elasto-plastic response of TZP (tetragonal zirconia polycrystal, $90 \%$ tetragonal phase) to the stresses introduced by indentation. The damage inflicted on this material which is prone to stress-induced phase transformation and plasticity at room temperature, is studied here by observing the changes in the load bearing capacity with normal load and initiation of crack modes, especially the additional modes believed to be initiated due to phase transformation. The usefulness of such a study lies in interpreting data obtained from impact erosion and abrasive wear.

Cook and Pharr (1990) and Yoffe (1982) studied the indentation fracture behaviour of soda lime glass and other ceramics by employing elasto-plastic continuum analysis. They predict the initiation of a crack type by observing the directionality of the tensile stress. As transformation causes $4 \%$ volume expansion and local damage, often in the form of transformation pits in the transformed volume, the stress field in this volume cannot be determined easily using continuum theories of elasticity and plasticity. It is for this reason that the present problem is addressed in two stages. The material is first idealized such that its response to indentation is purely elastic involving no phase transformation. An elastic stress field is used at this stage to identify the possible crack types which will be initiated given the 
elastic constants of TZP. The cracks observed experimentally in addition to these types are then stated to be due to phase transformation. The possible reason for the generation of these additional cracks is discussed.

In comparing the competence of the three proposed (Chiang et al 1982; Yoffe 1982; Johnson 1985) stress fields in elasto-plastic indentation of copper, Ghosal and Biswas (1992) have recently found Yoffe's stress field to be the most competent one in predicting the surface strain distribution. Mukhopadhyay and Biswas (1991) and Cook and Pharr (1990) found Yoffe's stress field useful in describing mechanical response of brittle materials to indentation. In the present work Yoffe's stress field is used to describe the nature of stresses generated by indentation in the TZP subsurface.

\section{The stress distribution}

The elastic stress field proposed by Yoffe consists of two components; a Boussinesq field dependent on applied normal load and varying inversely with the square of the distance and a blister field originally due to Love (1920) determined by the extent of permanent deformation. The stresses due to the latter vary inversely with the cube of the distance. The stress components in polar $(r, \theta, \phi)$ coordinates are,

$$
\begin{aligned}
& \sigma_{r}=\left(L / 2 \pi r^{2}\right)[1-2 v-2(2-v) \cos \theta]+4\left(B / r^{3}\right)\left[(5-v) \cos ^{2} \theta-(2-v)\right], \\
& \sigma_{\theta}=\left(L / 2 \pi r^{2}\right)\left[(1-2 v) \cos ^{2} \theta / 1+\cos \theta\right]-2\left(B / r^{3}\right)(1-2 v) \cos ^{2} \theta, \\
& \sigma_{0}=L(1-2 v) / 2 \pi r^{2}[\cos \theta-(1 / 1+\cos \theta)]+2\left(B / r^{3}\right)(1-2 v)\left(2-3 \cos ^{2} \theta\right), \\
& \tau_{r \theta}=\left(L(1-2 v) / 2 \pi r^{2}\right)(\sin \theta \cos \theta / 1+\cos \theta)+4\left(B / r^{3}\right)(1+v) \sin \theta \cos \theta,
\end{aligned}
$$

where $L$ is the applied load, $v$ the Poisson's ratio, and $B$ the blister field strength expressed in units of energy.

Cook and Pharr (1990) estimates the parameter $B$ from volume considerations. The volume swept by any hemisphere of radius $r$ measured from the point of indentation may be found by applying Hooke's law to the stress field given by (1):

$$
\Delta v=2 \pi B(1-2 v) / 3 G
$$

where $G$ is the shear modulus.

Now the volume displaced by a conical indenter of included angle $2 \alpha$ is

$$
\Delta v=\Pi h_{r}^{3} \tan ^{2} \alpha / 3,
$$

where $h_{r}$ is the residual indentation depth.

Equating (1) and (2) gives

$$
B=f\left[E h_{r}^{3} \tan ^{2} \alpha / 4(1-v)(1-2 v)\right]
$$


where $E$ is the Young's modulus and $f$ the densification factor. Cook and Pharr (1990) note that the densification factor for a commercially compacted and sintered ceramic is 1 . With reference to (1), the parameter $B$ is seen to be directly related to the residual stress $(L=0)$ and it has the units of energy. Lawn and Howes (1983) associated a permanent indent in elastic-plastic indentation with a residual energy analogous to that stored in a pre-compressed spring. On release of this residual energy the indentor depth is reduced to zero. If this concept is correct as is demonstrated by Lawn and Howes (1983), it is likely that the parameter $B$ is directly associated with such a residual energy deposited in an indent.

\section{Experimental}

The test material is tetragonal zirconia polycrystals (TZP, 80\% tetragonal phase) of density $6.05 \mathrm{~g} / \mathrm{cm}^{3}$, Vicker's hardness $13 \mathrm{Gpa}$, Young's modulus $205 \mathrm{Gpa}$ and Poisson's ratio $0 \cdot 3$. Cylindrical specimens of $5 \mathrm{~mm}$ dia and $5 \mathrm{~mm}$ length specimens were cut using a low speed diamond saw from a commercially obtained $5 \mathrm{~mm}$ dia TZP rods. The as cut surface of roughness $0.1 \mu \mathrm{m}$ CLA was indented using diamond conical indenters of cone angle and $150^{\circ}$. The indentation was carried out in quasi static mode. The specimen was placed on a (5 kN maximum) load cell. A schematic of the indentation rig is given in figure 1. The displacement of the load cell due to $4 \mathrm{kN}$ (maximum experimental load) compressive load is $\approx 4 \mu \mathrm{m}$. Considering that this order of displacement is very small compared to the indentation depths of the order of $160 \mu \mathrm{m}$ measured at this order of load, the reported penetration depth can be taken to be a reasonable representation of the true penetration depth. The load $L$ was continuously measured during experiment as a function of the penetration depth $h$ which was measured by an LVDT placed on a rigid platen

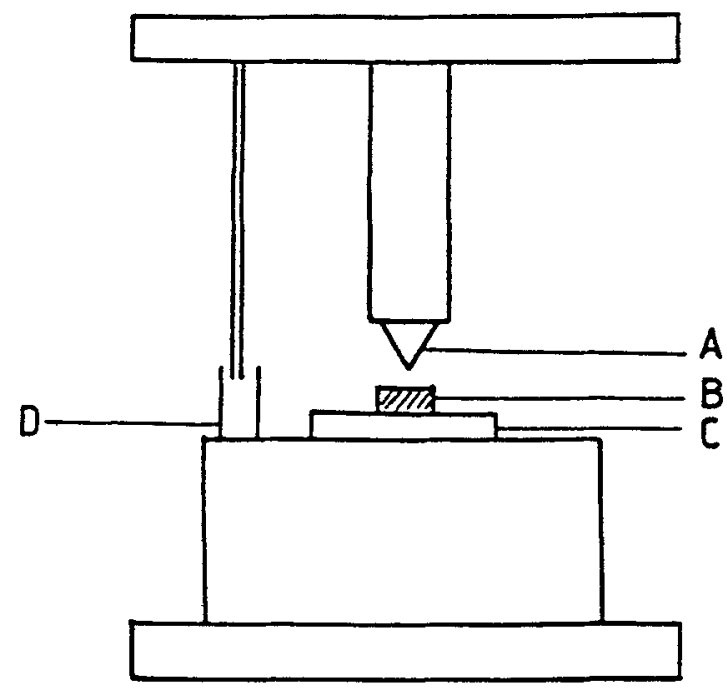

Figure 1. A schematic of the indentation set up (A, indenter; B, specimen; C, load cell; D, LVDT). 
which also supported the load cell. The whole assembly was placed inside a precision subpress which was driven hydraulically in a universal testing machine. The surface zone and the diameteral cross-section of the subsurface zone were examined by SEM and optical microscopy.

\section{Results}

\subsection{Influence of transformation on load-penetration characteristics}

Loubet (1986) gives the load-penetration relation in conical indentation of elasto-plastic material as

$$
P=K h^{2}
$$

where $h$ is the depth of penetration and $K$ a constant given by

$$
\left.K=\left[\left(1 / \Pi H \tan ^{2} \alpha\right)^{1 / 2}\right)+\left|\left(2\left(1-v^{2}\right) / \Pi E \tan \alpha\right)\right|^{1 / 2}\right]^{-2} .
$$

Table 1 gives the hardness of cubic, tetragonal and monoclinic phases of zirconia. The Young's modulus is structure insensitive. Figure 2 shows the theoretical

Table 1. Hardness and Young's modulus of zirconia.

\begin{tabular}{lccl}
\hline Constituent & $H(\mathrm{GPa})$ & $E(\mathrm{GPa})$ & \multicolumn{1}{c}{ References } \\
\hline Cubic & 15.5 & & Chen and Reyes-Morel (1986) \\
Tetragonal & 13 & 205 & $\begin{array}{l}\text { Test material } \\
\text { Monoclinic }\end{array}$ \\
\hline
\end{tabular}

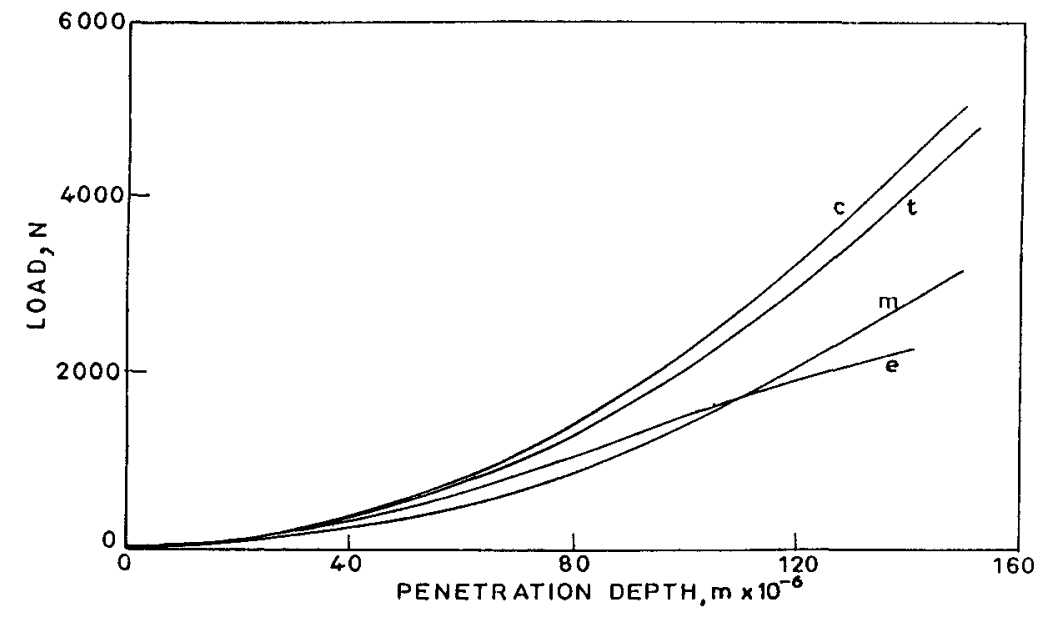

ligurc 2. Load-penetration in indentation by a $150^{\circ}$ diamond cone. Predicted characteristic for monophase zirconia (c, Cubic; $t$, tetragonal; $m$, monoclinic; e, experimental characteristics for tetragonal zirconia polycrystals). 

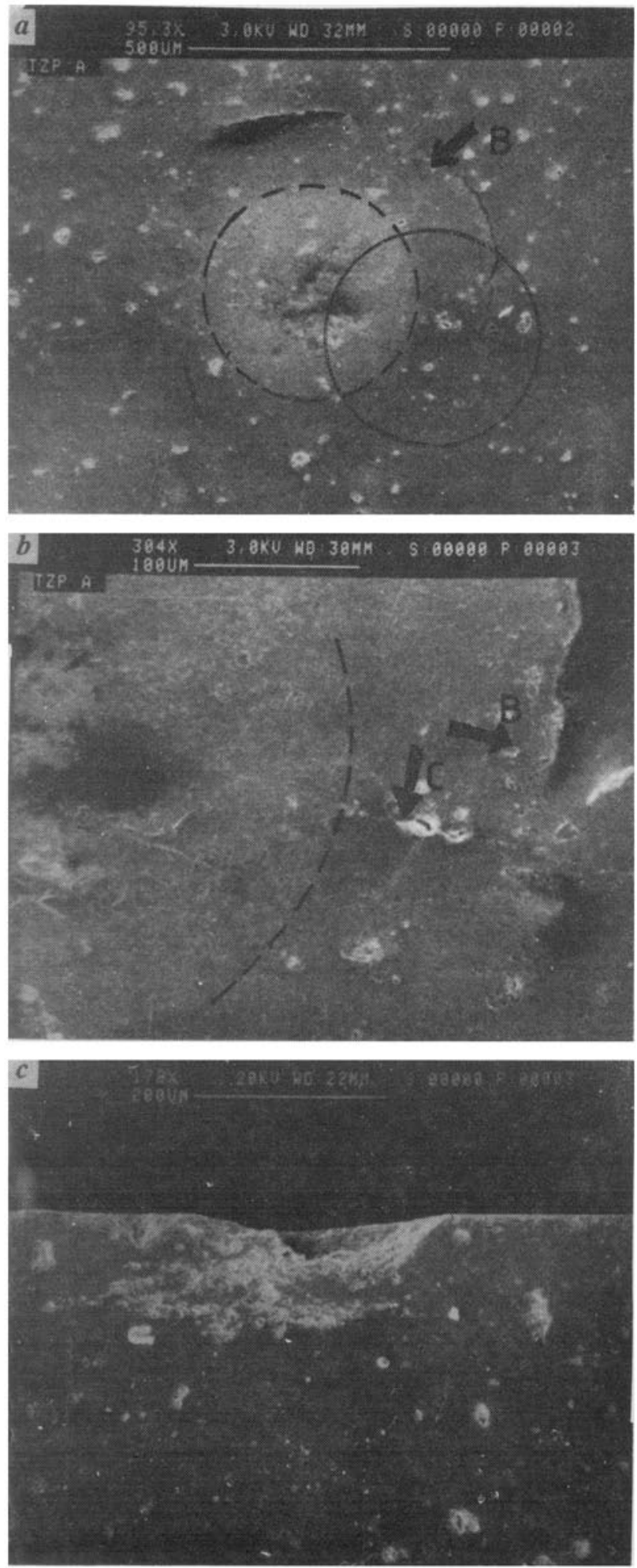

Figure 3a-c. For caption, see page 869. 

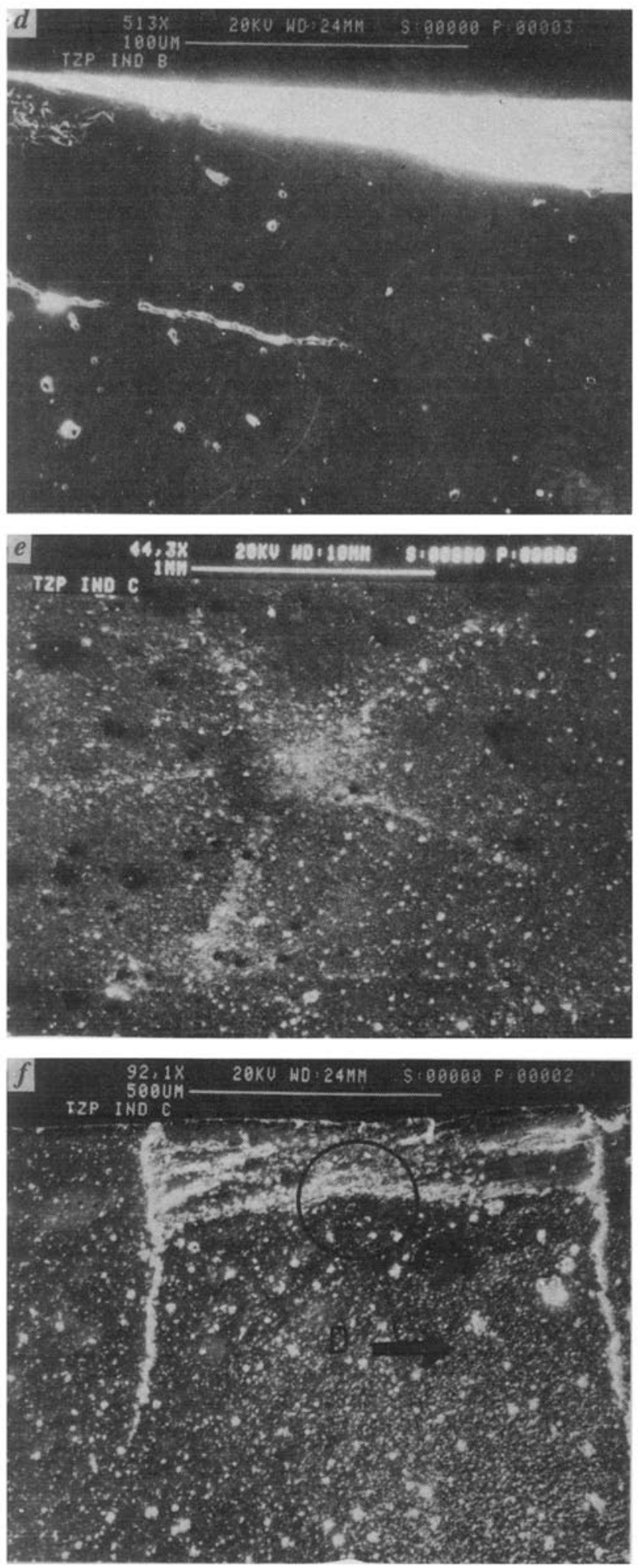

rigure Sd-f. For caption, see page 869. 


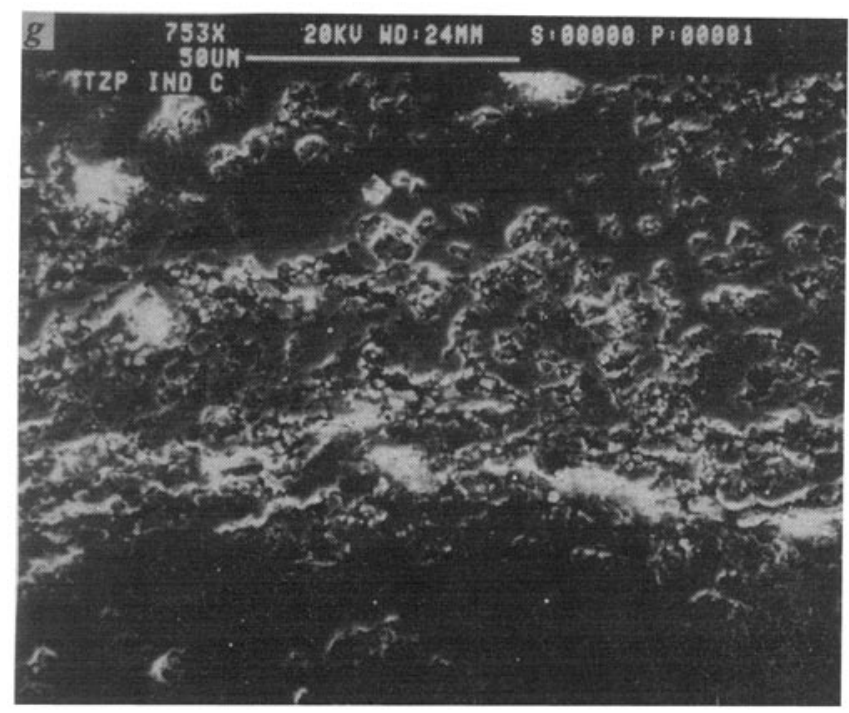

Figure 3. a-g. a. Surface SEM micrograph of $150^{\circ}$ conical indentation loaded to $1900 \mathrm{~N}$ showing ring and radial cracks: $(--)$ periphery of the indent; $(B)$ ring crack, b. magnified view of the circled region of figure $3 a$ showing radial (marked $C$ ) and ring (marked B) cracks, c. subsurface SEM micrograph of $150^{\circ}$ conical indentation loaded to $1900 \mathrm{~N}$, showing deformed zone, d. subsurface micrograph of $150^{\circ}$ conical indentation loaded to $1900 \mathrm{~N}$, showing lateral cracks, e. surface micrograph of $150^{\circ}$ conical indentation loaded to $3750 \mathrm{~N}$, showing radial cracks with heavily pitted zone, f. subsurface micrograph of $150^{\circ}$ conical indentation loaded to $3750 \mathrm{~N}$, showing median (marked D), cone and lateral cracks with heavily pitted zone and g. a magnified view of the circled region of $f$ showing heavily pitted and disturbed region left in the wake of lateral crack.

load-penetration curves for all the three monophase materials estimated using (4).

Figure 2 shows that at loads below $1000 \mathrm{~N}$ the experimental load-penetration curve is in close agreement with that of the theoretical load-penetration curve of the tetragonal monophase material. At loads higher than $1000 \mathrm{~N}$ the experimental curve deviates significantly from the tetragonal curve with increasing load and tends towards the monoclinic. At higher loads (above $1900 \mathrm{~N}$ ) the theoretical monoclinic curve overestimates the experimental results. This is indeed possible if there is a transformation of the original (85\%) tetragonal phase during loading to a low load bearing monoclinic phase, and accumulation of contact mechanical damage.

\subsection{Fracture behaviour}

The fracture behaviour of TZP is studied here by indenting it with a $150^{\circ}$ included angle cone loaded to a maximum load of $3750 \mathrm{~N}$. The surface containing the indent and the cross-section of the indented block (containing a diametral section of the indent) are viewed in SEM to observe the cracks corresponding to the two indentation loads (figure 3 ). To study the fracture behaviour, the elastic stresses in the subsurface are computed using (1) and the nature of the three orthogonal stresses $\left(\sigma_{\phi}, \sigma_{r}, \sigma_{\theta}\right)$ 

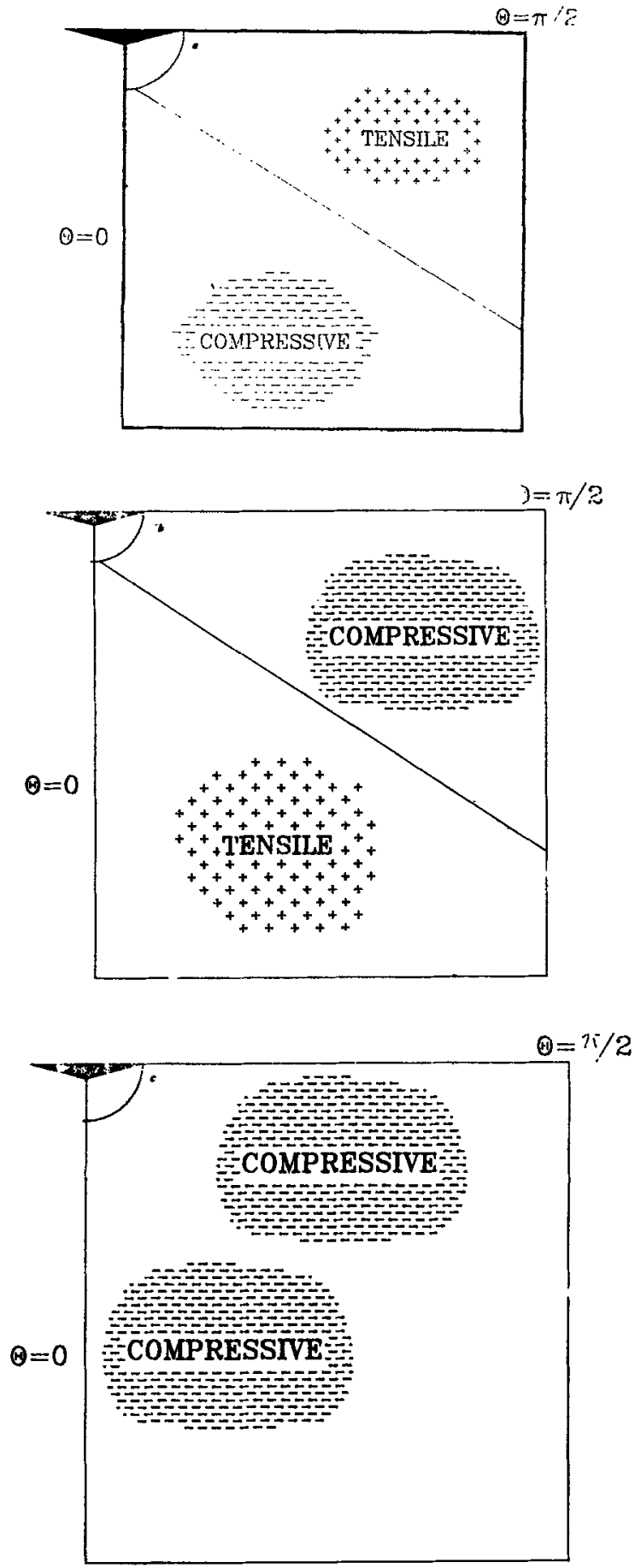

Figure 4. Indentation stress field as per (1). a. $\sigma_{\phi}$, hoop stress; b. $\sigma_{r}$, radial stress; c. $\sigma_{\theta}$, tangential stress. 
is shown in figure 4. Considering that the stresses in the blister are highly compressive figure $4 a$ indicates the possibility of a radial crack initiating at the edge of the contact where the tensile hoop $\left(\sigma_{\phi}\right)$ stress is maximum. Figure $4 \mathrm{c}$ indicates the possibility of lateral crack initiation $\left(\sigma_{r}\right.$ is tensile). Experimental observations, especially the fact that the radial crack is initiated at the contact edge (figure $3 \mathrm{~b}$ ), support the above predictions. It has to be noted that the nature of the subsurface stress field as shown in figure 4 do not change with the normal load. For the present material as the blister field is dominant over Boussinesq fields, (1) gives that the nature of $\sigma$, and $\sigma_{\phi}$ do not also change on unloading $(L=0)$ but the stresses become more accentuated on unloading. Unloading thus cannot cause any new crack type to appear but it may help to propagate the already existing radial and lateral cracks or the radial and lateral cracks may become more numerous. Table 2 summarizes experimental observations and the theoretical predictions of crack initiation.

Figure 3 and table 2 show the presence of additional cracks; ring crack at low loads and ring, median and cone cracks at high loads, cracks which are not accounted for in the model behaviour of the idealized elastic material. These additional cracks may arise due to the different crack types interacting energetically. Cook and Pharr (1990) attributed the appearance of the secondary radial cracks in the indented anomalous soda lime glass to the release of residual energy caused by the nucleation and propagation of primary cone cracks.

Figures $3 b-f$ show that radial and lateral cracks when they propagate leave in their wake heavily pitted and disturbed regions. Figure $3 \mathrm{~g}$ shows a magnified view of such a region left in the wake of a lateral crack. Reece et al (1992) used Raman microprobe to identify such pitted regions as regions which have undergone phase transformation from tetragonal to monoclinic. They ascribe the pitting to the reduced bonding between adjacent grains because of microcracking produced during phase transformation. Such pitting is likely to reduce the residual energy (directly associated with $B$ in $\$ 2$ ) and open up the possibility of secondary crack types. Figure 5 shows the variation of stresses, computed using (1), with $B$. Figure 5 a shows that if the material damage at the low load $(1900 \mathrm{~N})$ reduces the $B$ values

Table 2. Indentation cracking in zirconia.

\begin{tabular}{|c|c|c|c|c|}
\hline \multirow[b]{2}{*}{ Cracks } & \multicolumn{2}{|c|}{ Load I $(1900 N)$} & \multicolumn{2}{|c|}{ Load $2(3750 N)$} \\
\hline & Exp. & Theor. & Exp. & Theor. \\
\hline Radial & $\begin{array}{c}Y \\
\text { (figure } 3 b \text { ) }\end{array}$ & $\begin{array}{c}\mathrm{Y} \\
\text { (figure 4a) }\end{array}$ & $\begin{array}{c}\mathrm{Y} \\
\text { (figure } 3 \mathrm{e} \text { ) }\end{array}$ & $\begin{array}{c}Y \\
\text { (figure 4a) }\end{array}$ \\
\hline Ring & $\begin{array}{c}\mathrm{Y} \\
\text { (figure 3a) }\end{array}$ & $\stackrel{N}{\text { (figure } 4 b)}$ & $\begin{array}{c}\mathrm{Y} \\
\text { (figure } 3 f \text { ) }\end{array}$ & $\stackrel{N}{\text { (figure } 4 b \text { ) }}$ \\
\hline Lateral & $\begin{array}{c}Y \\
\text { (figure 3d) }\end{array}$ & $\begin{array}{c}Y \\
\text { (figure 4b) }\end{array}$ & $\begin{array}{c}Y \\
\text { (figure 3f) }\end{array}$ & $\begin{array}{c}Y \\
\text { (figure 4b) }\end{array}$ \\
\hline Median & $\stackrel{\mathrm{N}}{\text { (figure } 3 \mathrm{c} \text { ) }}$ & $\begin{array}{c}\mathrm{N} \\
\text { (figure 4c) }\end{array}$ & $\begin{array}{c}Y \\
\text { (figure } 3 f \text { ) }\end{array}$ & $\frac{N}{\text { (figure } 4 c \text { ) }}$ \\
\hline Conical & $\stackrel{N}{\text { (figure } 3 c \text { ) }}$ & $\frac{N}{\text { (figure } 4 b \text { ) }}$ & $\begin{array}{c}Y \\
\text { (figure 3f) }\end{array}$ & $\begin{array}{c}\mathrm{N} \\
\text { (figure 4b) }\end{array}$ \\
\hline
\end{tabular}

Y, Yes; N, No. 

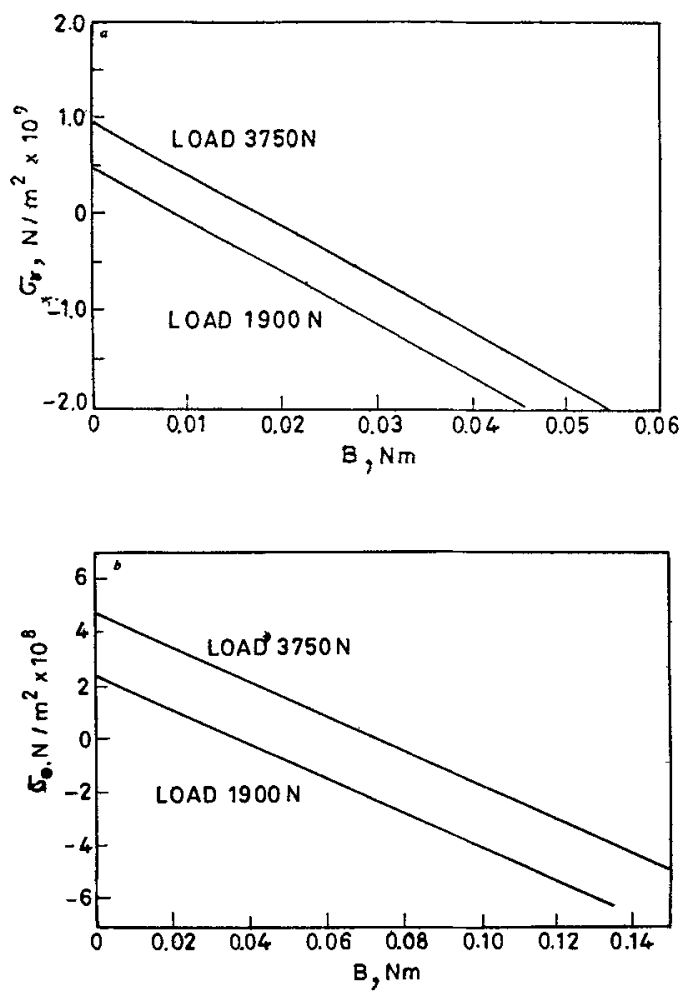

Figure 5. Variation of stresses with B. a. $\sigma_{r}$ at $r=500 \mu \mathrm{m}, \theta=90^{\circ}$ and b. $\sigma_{\theta}$ at $r=500 \mu \mathrm{m}, \theta=0^{\circ}$.

from $0.134 \mathrm{Nm}$ (calculated using (3)) to $0.01 \mathrm{Nm}$, the $\sigma_{r}$ becomes tensile, creating the possibility of a ring crack initiation at low load. While less material damage $(B-0.36$ to $0.05 \mathrm{Nm})$ is required (figure $5 \mathrm{~b}$ ) to initiate a median crack at this load $(3750 \mathrm{~N})$, the order of $\sigma_{\theta}\left(\theta=0^{\circ}\right)$ may be too low (half that of the corresponding $\sigma_{r}$, at $B=0$ ) for that to happen. It is considered that with an increase in normal load the extent of material damage due to phase transformation in the subsurface increases and the subsurface stresses become more and more tensile. At high load the ring crack at the surface may propagate into the subsurface to give rise to a cone crack. Figure $5 \mathrm{~b}$ shows that $\sigma_{\theta}\left(\theta=0^{\circ}\right)$ at this load becomes tensile at about $0.08 \mathrm{Nm}$ and the level of the stress is also doubled (at $B=0$ ) due to the load increment. Increment in load would thus tend to encourage the initiation of a median crack. With increasing load more crack types thus come into existence and the load bearing capacity becomes increasingly less than that of a purely monoclinic material at high loads, as seen in figure 2 .

\section{Conclusions}

3Y-TZP blocks were indented by conical diamond indenters. The material underwent phase transformation from tetragonal to monoclinic leaving highly pitted and disturbed 
region in the transformed subsurface. An attempt was made to separate the crack initiation due to a purely elastic response of the material from that due to phase transformation. A combination of near field stress regime due to a blister which account for the permanently deformed material and a far field regime due to the Boussinesq point force was used to describe the elastic stress field due to indentation. At low loads the radial, lateral and ring cracks and at high loads additional lateral, median and cone cracks were observed.

A purely elastic response of TZP to indentation can initiate radial and lateral cracks. The material damage caused by phase transformation can initiate a ring crack at low loads by lowering the residual energy. As the load is increased the damage becomes extensive paving the way for an extension of the ring crack to a cone crack and the initiation of a median crack.

This progressive weakening of the material caused by an increase in indentation load is considered to be responsible for the corresponding reduction in load bearing capacity.

\section{Acknowledgement}

The authors are grateful to the Department of Science and Technology, New Delhi for financial support.

\section{References}

Chen I W and Reyes-Morel P E $1986 \mathrm{~J}$. Am. Ceram. Soc. 69 181, 189

Chiang S S, Marshall D B and Evans A G 1982 J. Appl. Phys. 53298

Cook R F and Pharr G M 1990 J. Am. Ceram. Soc. 73787

Ghosal A K and Biswas S K 1992 Philos. Mag. 67371

Johnson K L 1985 Contact mechanics (Cambridge University Press)

Lawn B R and Howes V R 1983 J. Mater. Sci. 162745

Loubet J L 1986 Microindentation techniques in materials science and engineering (eds) P Blau and B R Lawn (Philadelphia, Pennsylvania: ASTM STP 889)

Love A E H 1920 The mathematical theory of elasticity (Cambridge University Press)

Marshall D B and James M R $1986 \mathrm{~J}$. Am. Ceram. Soc. 69215

Martinez-Fernandez J, Jimenez-Melendo M, Dominguez-Rodriguez A and Heuer A H $1991 \mathrm{~J}$. Am. Ceram. Soc. 741071

Mukhopadhyay A and Biswas S K 1991 Philos. Mag. Lett. 6477

Reece M J, Tetlow P L and Galiotis C 1992 J. Mater. Sci. Lett. 11575

Yoffe E H 1982 Philos. Mag. A46 617 